\title{
The Lehigh SEM, AEM and SPM Short Courses - 1993
}

\section{Basic Course: Scanning Electron Microscopy and X-ray Microanalysis (June 14-18, 1993)}

A basic course directed toward academic, industrial, and governmental scientists, engineers, biologists, technicians, and technical managers whose activities and interests relate to SEM and $\mathrm{x}$-ray microanalysis. The course has two options: Materials and Biology/Polymers, with lecture and laboratory topics appropriate to each. Materials Course: electron beam specimen interactions, high resolution SEM, $\mathrm{x}$-ray detectors, quantitative $\mathrm{x}$-ray analysis, SEM characterization of electronic materials, environmental SEM techniques, digital image analysis. Biology Course: ambient temperature/low temperature specimen preparation, SEM of polymers and fibers, immunocytochemical methods, clinical applications of SEM/X-ray microanalysis. Seven SEM's, one automated electron microprobe, and various EDS/WDS instruments will be available for the labs and for hands-on use later in the course. A textbook (new edition 1992) and a lab workbook written by the instructors of the course will be provided to all registrants.

\section{Advanced Courses:}

\section{(June 21-25, 1993)}

These courses are directed toward academic, industrial, and governmental scientists, engineers, advanced technicians, and technical managers who want in-depth study in the following areas. Seven SEM's and one automated electron microprobe with EDS/WDS $x$-ray capability as well as several STM instruments will be available in the laboratory sessions.

\section{Microcharacterization of Electronic Materlals, Devices and Packages}

This course covers the physical principles and practical aspects of the following subject material: voltage contrast, low voltage microscopy, charge collection microscopy, cathodoluminescence, scanning tunneling microscopy, confocal optical microscopy, and surface analysis (SIMS, Auger, XPS).

\section{Advanced Scanning Imaging}

This course is directed towards SEM users who wish to obtain the highest possible resolution from their SEM. Lecture and laboratory topics include: high resolution SEM (low-loss BSE, STEM in SEM, and SE-I/ SE-II Imaging), advanced coating techniques, low-voltage microscopy, environmental SEM, computer-aided imaging, framestore real time digital imaging, quantitative stereo, scanning tunneling and atomic force microscopy.

\section{Quantitative X-ray Microanalysis of Bulk Specimens and Particles}

SEM or microprobe operators and supervisors who wish to advance their knowledge of $x$-ray microanalysis will find this course of interest. Topics include: $x$-ray production; ZAF calculations using PCs; WDS/EDS detectors; quantitative analysis of particles and rough specimens, computer-aided imaging, error analysis and statistics, strategy for applying microanalysis techniques and light element analysis.

\section{AFM, STM and Other Scanned Probe Microscoples}

This course is in its second year and was very popular in its initial year. This course will cover the physical properties and practical aspects of the following subject material: scanning tunneling microscopy, atomic force microscopy, scanning tunneling spectroscopy, scanning tunneling potentiometry, feedback control, tip fabrication, scan calibrations, in-situ imaging, UHV imaging, imaging in air, image processing, near field optical probes, surface science applications, metrology, engineering applications, lateral force microscopy, electrochemical STM/AFM, and other emerging scanned probe techniques.

\section{Analytical Electron Microscopy (June 21-24, 1993)}

This course covers the range of analytical techniques available on modern TEM/STEM and dedicated STEM instruments. Lectures and labs cover STEM optics and instrumentation, Z-contrast and other STEM imaging modes, convergent beam electron diffraction, and quantitative microanalysis using $\mathrm{x}$-ray EDS and EELS.

\section{Thin Specimen Preparation (June 24-25, 1993)}

This course is directed toward all AEM and TEM users who want to learn the latest techniques of thin specimen preparation. Basic principles and practical laboratory procedures will be illustrated. Topics include: initial thinning, electropolishing, ion-beam milling, ultramicrotomy, techniques for cross-sections of interfaces, particles, etc.

For detailed course brochures and registration forms, please contact Professor Joseph I. Goldstein, Department of Materials Science and Engineering, 5 E. Packer Avenue, Lehigh University, Bethlehem, PA 18015.

Phone: $215 / 758-5133$, FAX: 215/758-4244 